\title{
Some New Properties of the Dilute Polymer Solutions I. Excluded Volume and Interpenetration of Macromolecules in Solutions
}

\author{
V. E. ESKIN, I. A. BARANOVSKAYA, and U. S. KHUDAIBERDIEV \\ Institute of Macromolecular Compounds of the Academy of \\ Sciences of the U.S.S.R., Leningrad, U.S.S.R.
}

(Received January 29, 1986)

\begin{abstract}
Light scattering was investigated for polystyrene over a wide molecular weight range in solvents of various thermodynamic strength in the concentration range lower and higher than $(100 / M)$. In all cases, the slope of the plots $c K / R_{\theta}=f(c)$ at $c<100 / M$ was close to horizontal. The concentration $c_{0}$ in the range in which the slope of the plot $c K / R_{\theta}=f(c)$ passes from the quasihorizontal to the "usual" vertical slope, is determined only by the molecular weight of the polymer and is virtually independent of the solvent. The excluded volume $b$ and energy of attraction $a$ of macromolecules are determined by using the two-parameter Van der Waals equation. The $b_{0} / V_{\mathbf{M}}$ ratio of the molar values of the excluded volume and the volume proper of macromolecules allows conclusions to be drawn about the degree of interpenetration of the macromolecules and its dependence on the molecular weight and the strength of the solvent. A relationship is established between the swelling coefficient $\alpha \eta$ of coils and the density $\rho$ of segments in center of the zone of interpenetration of two macromolecules.
\end{abstract}

KEY WORDS Very Dilute Polymer Solutions / Thermodynamic Properties / Light Scattering / Virial Coefficient / Interpenetration of Molecular Coils /

In 1977, we reported ${ }^{1}$ a reproducible "anomalous" property of polystyrene solutions in benzene: the inverse scattering intensity $c K / R_{\theta}$ was independent, within experimental error, on the concentration below a certain concentration $c_{0 l} \cdot{ }^{* 1}$ In this case, the dependence of the "threshold" concentration on the molecular weight of the polymer (at $\bar{M}_{w} \lesssim 5 \times 10^{5}$ ) had the form $c_{0 l} \sim \bar{M}_{w}^{-0.8}$. More recently, similar behavior has been observed for polystyrene in other solvents as well as for other polymers ${ }^{3,4 a}$ (Figure 1). The curves, of asymmetry of light scattering $(Z-1)^{-1}$ are constant at sufficiently low concentrations (Figure 2). Here $Z=R_{45^{\circ}} / R_{135^{\circ}}$. This is in agreement with the well-known Zimm's equa- tion $^{5}$

$$
(z-1)^{-1}=([z]-1)^{-1}\left(1+2 A_{2} M P\left(45^{\circ}\right) c\right)
$$

(where $[z]$ is the characteristic asymmetry of scattering at $c \rightarrow 0$ and $A_{2}$ is the second virial coefficient of solution) only at $A_{2} \simeq 0$.

The shape of the light scattering plots for polymer solutions in thermodynamically good solvents similar to that shown in Figures 1 and 2 cannot be treated in the terms of traditional interpretation. ${ }^{6,7}$

Since the curves $\pi / c$ of osmotic pressure of polymer solutions and hence of their light scattering cannot have breaks, we suggest ${ }^{8}$ that the quasihorizontal plot $c K / R_{\theta}=f(c)$ is actually a very slightly concave curve in the

*1 A similar phenomenon has previously been observed by the authors for solutions of a graft copolymer of styrene and methyl methacrylate with the molecular weight $\bar{M}_{w}=5.5 \times 10^{6} .^{2}$ 

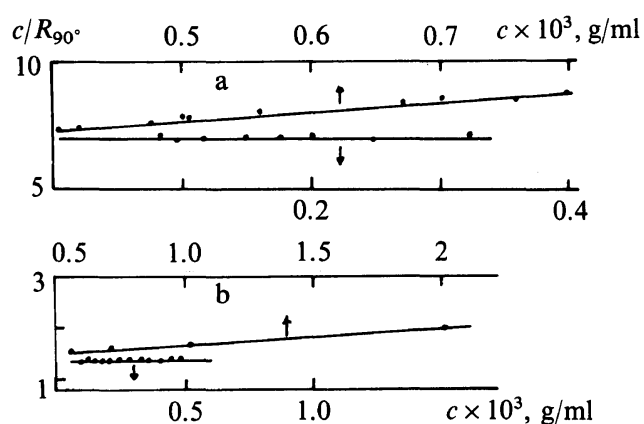

Figure 1. Initial and subsequent courses of experimental light scattering plots $c / R_{90^{\circ}}=f(c)$ for a) PS in benzene, $\bar{M}_{w}=3.1 \times 10^{6}$ and b) poly(naphthyl methacrylate) in 1,2-dichloroethane, $\bar{M}_{w}=1.6 \times 10^{6}$.

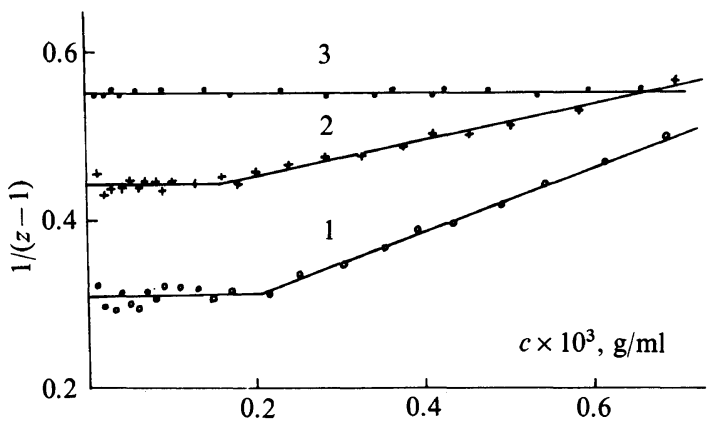

Figure 2. Concentration dependence of $(z-1)^{-1}$ for PS with $\bar{M}_{w}=5.5 \times 10^{6}$ in a) benzene, 2) methyl ethyl ketone, 3) $\theta$-solvent.

range $c<c_{0 l}$. This plot seems to be horizontal only because of the spread of experimental points masking its curvature.

In this case, we proceed from the wellknown Einstein equation ${ }^{9}$ for the Rayleigh ratio. $R_{\theta}$ of light scattered by a solution of particles small compared to the light wavelength at a concentration $c$ is

$$
R_{\theta}=\frac{c K}{\partial \pi / \partial c}
$$

It is possible to obtain

$$
\frac{c K}{R_{\theta}}=\frac{M}{(M-c b)^{2}}-\frac{2 c a}{M^{2} R T}
$$

where $M$ is the molecular weight of the polymer, $R$ is the universal gas constant, $T$ is the absolute temperature, $b$, is the excluded volume of a mole of macromolecules and $a$ is the energy of their mutual attraction.

The relationship between $a$ and $b$ may be established on the basis of general considerations applied by Debge ${ }^{10}$ to the study of critical opalescence of polymer solutions. According to ref 10 the energy of interaction (attraction) between two macromolecules is given by*2

$$
E=w \frac{p^{2}}{2(2 \pi)^{3 / 2}}\left(3 / 2\left\langle R^{2}\right\rangle\right)^{3 / 2} \exp \left(-3 r / 4\left\langle R^{2}\right\rangle\right)
$$

where $w$ is the energy of interaction of two units among their number $p$ in each molecule integrated over the volume, $r$ is the distance between the centers of molecules and $\left\langle R^{2}\right\rangle^{1 / 2}$ is their mean radius of gyration. The integration of the attraction energy $E$ for completely or partially impermeable coils should be carried out in the range from $r_{\mathrm{ex}}$ to infinity where $r_{\mathrm{ex}}$ is the radius of excluded volume of the approaching particles. When $r_{\mathrm{ex}} \neq 0$, the integral $\int E \mathrm{~d} v$ reduces to the incomplete gamma-function

$$
\Gamma\left(\frac{3}{2}, K\right)=\int_{n}^{\infty} \exp (-t) t^{(3 / 2)-1} \mathrm{~d} t
$$

where

$$
\begin{aligned}
& t=\frac{3}{4} r^{2} /\left\langle R^{2}\right\rangle \\
& K=\frac{3}{4} r_{\mathrm{ex}}^{2} /\left\langle R^{2}\right\rangle
\end{aligned}
$$

and $r$ is the distance between the centres of macromolecules.

In some parts of the plot, the dependence

$$
\Gamma\left(\frac{3}{2}, K\right)
$$

(3) on $K^{-3 / 2}$ is quite satisfactorily approximated by a straight line, and since

\footnotetext{
*2 In eq 4, the factor 2 was introduced into the denominator so as not to have to take into account twice the interaction between each pair of units belonging to two macromolecules.
} 
Table I. Values of $m$ and $n$ for the required ranges of the ratio of excluded volume $b$ to the volume proper of a mole of macromolecules

\begin{tabular}{lcl}
\hline \multicolumn{1}{r}{$x=b / V_{\mathrm{M}}$} & $m$ & \multicolumn{1}{c}{$n$} \\
\hline $2 \gtrless x \gtrless 5$ & 0.529 & -0.0687 \\
$4.1 \gtrless x \gtrless 6.8$ & 0.423 & -0.0455 \\
$5.8 \gtrless x \gtrless 9.0$ & 0.313 & -0.029 \\
\hline
\end{tabular}

$$
K^{-3 / 2}=0.608 V_{\mathrm{M}} / b
$$

where $V_{\mathrm{M}}$ is the volume proper of a mole of macromolecules (a homodisperse polymer sample is here considered), for definite ranges of values of $V_{\mathrm{M}} / b$, the coefficients $m$, and $n$ in the equation

$$
\Gamma\left(\frac{3}{2}, K\right)=m \frac{V_{\mathrm{M}}}{b}+n
$$

can be determined. The values of $m$ and $n$ for the required ranges of the ratio of excluded volume $b$ to the volume proper of one mole of macromolecules are given in Table I.

The attraction energy of units of two macromolecules $w p^{2} / 2$ contained in eq 4 may be expressed by the experimental value $\delta$ of the molecular cohesive energy density of the polymer

$$
w=\frac{2 \delta^{2} \bar{v}^{2} M_{0}^{2}}{N^{2}}
$$

where $\bar{v}$ is the partial specific volume of the polymer in a given solvent, $M_{0}$ is the molecular weight of a chain unit and $N$ is Avogadro's number. Since $p M_{0}=M$ one can obtain

$$
a=A(B / b+n)
$$

where

$$
\begin{aligned}
& A=0.282 M^{2}(\delta \bar{v})^{2} \\
& B=m M[\eta]
\end{aligned}
$$

if the volume proper of macromolecules is expressed by the intrinsic viscosity $[\eta]$ of the polymer.

At concentrations $c>c_{0 l}$, the light scat- tering of solutions satisfies the following linear dependence over a relatively wide (as compared to $c_{0 l}$ ) concentration range:

$$
c K / R_{\theta}=M^{-1}+2 A^{*}\left(c-c_{0 l}\right)
$$

For reasons following from part II of this publication, we will call $A^{*}$ the "effective" virial coefficient rather than the "seond" virial coefficient as is usually done. Eq 3 and $(11-11 b)$ yield the equation for the determination of $b$ :

$$
b^{3}+\frac{Y}{X} b^{2}+\frac{Z}{X} b+2 M^{2} A B / X=0
$$

where

$$
\begin{aligned}
X= & c M R T+2 c^{2} A n \\
& +2 c^{2} M^{2} R T A^{*}\left(1-c_{0 l} / c\right) \\
Y= & 2\left[c^{2} A B-2 c M A n\right. \\
& \left.-M^{2} R T-2 c M^{3} R T A^{*}\left(1-c_{0 l} / c\right)\right] \\
Z= & 2\left[M^{2} A n-2 c M A B\right. \\
& \left.+M^{4} R T A^{*}\left(1-c_{0 l} / c\right)\right]
\end{aligned}
$$

\section{MATERIALS AND METHODS}

Polystyrene was obtained by free-radical polymerization and fractionated to give 19 fractions by fractional precipitation with methanol from solution.

Light scattering was measured with a Sofica instrument in the angular range from 30 to $150^{\circ}$ at a temperature of $21^{\circ} \mathrm{C}$ in benzene, $20^{\circ} \mathrm{C}$ in methyl ethyl ketone and $24.5,34.5$, and $44.5^{\circ} \mathrm{C}$ in cyclohexane. Before measurements, the solutions were centrifuged for $1.5-2 \mathrm{~h}$ at $15-20$ thousand $\mathrm{rev} / \mathrm{min}$.

The refractive index increment, $\mathrm{d} n / \mathrm{d} c$, of the polymer in solutions was determined with a Pulfrich type refractometer with a differential cell and was 0.106 in benzene, 0.230 in methyl ethyl ketone and 0.170 in cyclohexane (at $34.5^{\circ} \mathrm{C}$ ). 
Table II. Data for polystyrene in various solvents

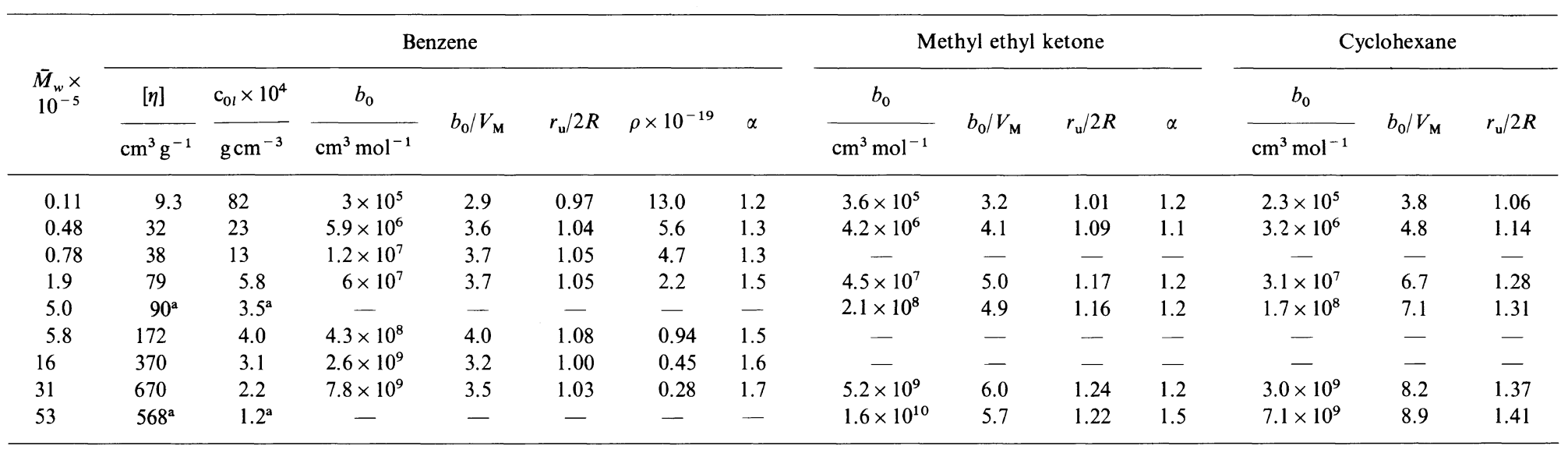

a Measured in methyl ethyl ketone. 
The solvents "pure for UV spectroscopy" were used.

\section{EXPERIMENTAL RESULTS AND DISCUSSION}

Equation 13 also makes it possible to obtain values of $b$ at concentrations $c \leqslant c_{01}{ }^{* 3}$ (It should be noted that at $c \leqslant c_{0}$ i.e., in the range of the quasihorizontal course of the scattering curve where $A^{*} \simeq 0$ the last term in eq $13 \mathrm{a}-13 \mathrm{c}$ is excluded). The values of $b_{0}$ obtained according to eq 13 and corresponding to $c=c_{0 l}$ as well as the $b_{0} / V_{\mathrm{M}}$ ratio $^{* 4}$ are listed in Table II. The molar value of the volume proper of macromolecules was calculated from the value of intrinsic viscosity. ${ }^{5}$

$$
V_{\mathrm{M}}=0.36 \frac{M[\eta]}{\Phi} N
$$

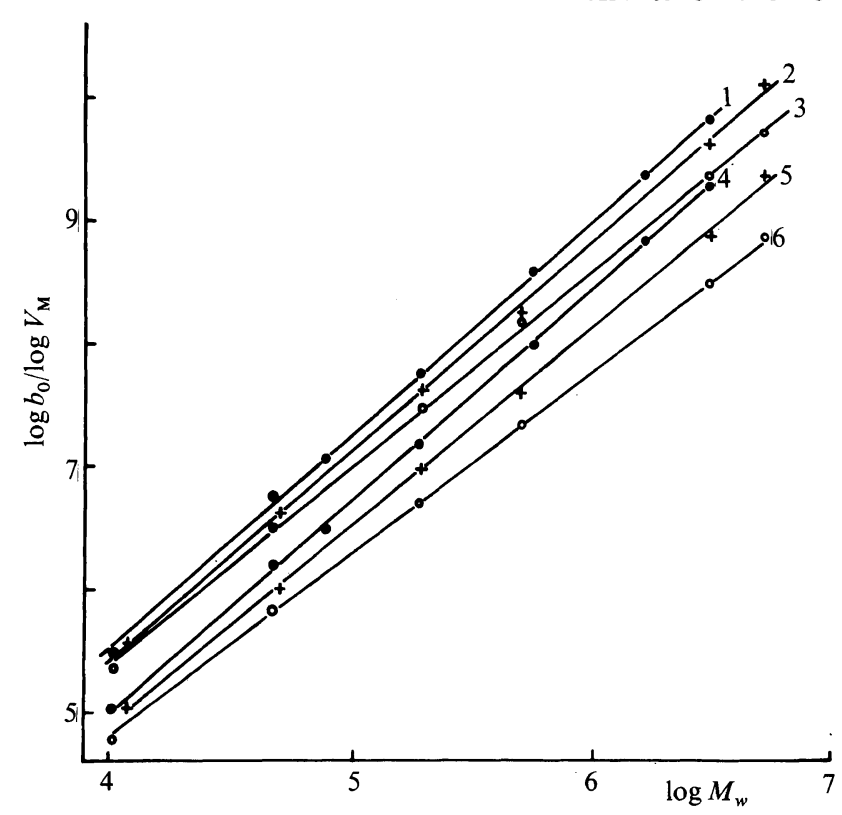

Figure 3. Excluded volume $(1-3)$ and volume proper $(4-6)$ of a mole of polystyrene macromolecules in benzene $(1,4)$ methyl ethyl ketone $(2,5)$ and cyclohexane at $\theta$-temperature $(3,6) v s$. molecular weight of the polymer; logarithmic scale.

*3 Equation 13 has an advantage over the equation for the osmotic pressure of solutions used by us in the above cited papers because it rules out the uncertainty present in the ratio of the "threshold" concentration $c_{0}$ to $c_{0 l}$ of the curves of osmotic pressure and light scattering.

${ }^{* 4}$ Since $c_{0 l}$ is slightly dependent on the solvent, $c_{0 l}$ for polystyrene in a $\theta$-solvent was assumed to be the average of values obtained in other solvents. 
Table III. Data for a polystyrene fraction with $\bar{M}_{w}=1.9 \times 10^{5}$ in five solvents

\begin{tabular}{|c|c|c|c|c|c|c|c|}
\hline \multirow{2}{*}{ Solvent } & {$[\eta]$} & \multirow{2}{*}{$\alpha$} & \multirow{2}{*}{$\frac{b_{0} \times 10^{-7}}{\mathrm{~cm}^{3} \mathrm{~mol}^{-1}}$} & \multirow{2}{*}{$b_{0} / V_{\mathrm{M}}$} & \multirow{2}{*}{$r_{\mathrm{u}} / 2 R$} & \multirow{2}{*}{$\frac{\rho \times 10^{-18}}{\mathrm{~cm}^{-3}}$} & \multirow{2}{*}{$\begin{array}{l}\rho \cdot \alpha \times \\
10^{-19}\end{array}$} \\
\hline & $\mathrm{cm}^{3} \mathrm{~g}^{-1}$ & & & & & & \\
\hline Benzene $\left(20^{\circ} \mathrm{C}\right)$ & 79 & 1.50 & 5.98 & 3.69 & 1.05 & 22.5 & 3.38 \\
\hline $\begin{array}{l}\text { Methyl ethyl ketone } \\
\qquad\left(20^{\circ} \mathrm{C}\right)\end{array}$ & 50 & 1.23 & 4.48 & 5.02 & 1.17 & 28.0 & 3.44 \\
\hline $\begin{array}{l}\text { Cyclohexane } \\
\qquad\left(\theta+10^{\circ} \mathrm{C}\right)\end{array}$ & 34 & 1.07 & 3.50 & 5.85 & 1.23 & 33.6 & 3.59 \\
\hline $\begin{array}{l}\text { Cyclohexane } \\
\qquad(\theta)\end{array}$ & 29 & 1.0 & 3.14 & 6.60 & 1.28 & 35.0 & 3.50 \\
\hline $\begin{array}{l}\text { Cyclohexane } \\
\qquad\left(\theta-10^{\circ} \mathrm{C}\right)\end{array}$ & 24.5 & 0.93 & 2.72 & 7.03 & 1.30 & 37.0 & 3.44 \\
\hline
\end{tabular}

more distinctly for one fraction $\left(\bar{M}_{w}=1.9 \times\right.$ $\left.10^{5}\right)$ in three solvents including cyclohexane at three temperatures (Table III) since in this case, additional errors in the determination of $\bar{M}_{w}$ and $c_{0 l}$ are excluded. The data given in Table III indicate that as the strength of the solvent decreases, the excluded volume $b_{0}$ decreases more slowly than the volume proper of macromolecules $V_{\mathbf{M}}$. As a result, the $b_{0} / V_{\mathrm{M}}$ ratio increases and consequently the ratio $r_{\mathrm{u}} / 2\left\langle R^{2}\right\rangle^{1 / 2}$ of the semiradius $\left(r_{\mathrm{u}} / 2\right)$ of the excluded volume (represented by a sphere) to the radius of gyration of the coil also increases (Figure 4).

Table III also contains the values of swelling coefficients $\alpha$ and density of polymeric units $\rho$ in the center of the overlapping zone of two coils at a distance $r_{u} / 2$ from the center of each coil. An important result is the constancy of the product $(\rho \alpha)$. The invariance of the value of $\rho \alpha$ (at $M=$ const) for a given polymer fraction in a series of solvents reflects a direct relationship between the processes of coil swelling and their interpenetration when they approach each other in solution.

On the basis of the empirical relationship obtained $(\rho \alpha)=P=(3.47 \pm 0.08) \times 10^{19}$, it is possible to establish the dependence of the degree of coil permeability $r_{u} / 2\left\langle R^{2}\right\rangle^{1 / 2}$ on the swelling coefficient $\alpha$. The density of segments

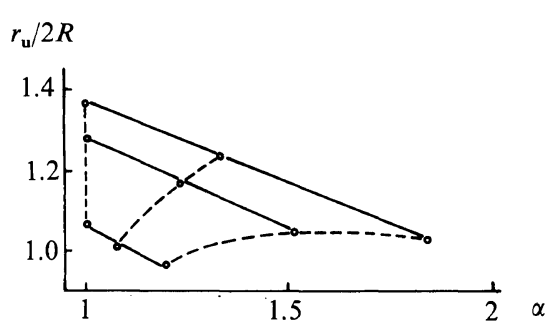

Figure 4. Relative permeability $\left(r_{u} / 2\left\langle R^{2}\right\rangle^{1 / 2}\right)$ of PS coils $v s$. swelling coefficient $\alpha$; solid curves, for different $M$ (from top to bottom, $3.1 \times 10^{6} ; 1.9 \times 10^{5} ; 1.05 \times 10^{4}$ ), broken curves, in different solvents (from left to right, cyclohexane at the $\theta$-point, methl ethyl ketone, benzene).

at a distance $r$ from the coil center is given by (see ref 7):

$$
\rho=0.33 N /\left\langle R_{0}^{2}\right\rangle^{3 / 2} \alpha^{2} \exp \left(\frac{3}{2} r_{u}^{2} /\left\langle R^{2}\right\rangle\right)
$$

where $N$ is the number of units in a macromolecule.

Since $\left\langle R^{2}\right\rangle^{1 / 2}=\alpha\left\langle R_{0}^{2}\right\rangle^{1 / 2}\left(\left\langle R_{0}^{2}\right\rangle^{1 / 2}\right.$ is the radius of gyration of the coil in a $\theta$-solvent), we have,

$$
P=0.33 N /\left\langle R_{0}^{2}\right\rangle^{3 / 2} \alpha^{2} \exp \left(\frac{3}{2} r_{u}^{2} /\left\langle R^{2}\right\rangle\right)
$$

to give

$$
r_{\mathrm{u}} / 2\left\langle R^{2}\right\rangle^{1 / 2}=\frac{1}{2}\left(\frac{2}{3} \ln \frac{0.33 N}{\alpha^{2}\left\langle R^{2}\right\rangle^{3 / 2} P}\right)^{1 / 2}
$$


In this case for $\bar{M}_{w}=1.9 \times 10^{5}$, we have $N=1.83 \times 10^{3},\left\langle R_{0}^{2}\right\rangle^{3 / 2}=1.5 \times 10^{-18} \mathrm{~cm}^{3}$, and $P=3.47 \times 10^{19}$ to give

$$
r_{\mathrm{u}} / 2\left\langle R^{2}\right\rangle^{1 / 2}=\frac{1}{2}\left(\frac{2}{3} \ln \frac{11.6}{\alpha^{2}}\right)^{1 / 2}
$$

When eq 16-17 are used, it should be borne in mind that the values of $N$ and $\left\langle R_{0}^{2}\right\rangle$ depend on the molecular weight of the polymer $M$, whereas the empirical constant $P$ also depends on the nature of the polymer. Thus, for a polystyrene with $\bar{M}_{w}=3.1 \times 10^{6}$ the value of $P=5.06 \times 10^{18}$ is obtained.

Hence, eq 16 represents the desired relationship between two closely related processes: the swelling of macromolecules $(\alpha)$ and the degree of interpenetration $\left(r_{u} / 2\left\langle R^{2}\right\rangle^{1 / 2}\right)$ in a given solvent. The loosening of the coil structure upon swelling in a thermodynamically good solvent facilitates their interpenetration. On the other hand, as the solvent strength decreases, the polymer-polymer contacts become preferable. This fact is manifested in the increasing segment density $\rho$ in the center of coil overlapping as the solvent strength decreases (Table III). This situation is demonstrated in Figure 5 where the points show the values of $\rho$ for the fraction with $\bar{M}_{w}=1.9 \times 10^{5}$ in several solvents. Figure 5 shows also that in a good solvent, the phenomenon of displacement of a considerable part of units to the periphery plays an important part in the relative value of coil permeability $\left(r_{\mathrm{u}} / 2\left\langle R^{2}\right\rangle^{1 / 2}\right)$ : the curve $\rho=$ $f(r / 2)$ is higher in benzene at $r / 2>1.4 \times 10^{-6}$ than in cyclohexane. However, the value of $\rho$ in the center of the zone of permeability remains higher in cyclohexane.

The effect of the length of chain molecules on their interpenetration explain the above mentioned dependence of the $b_{\mathrm{o}} / V_{\mathrm{M}}$ ratio (or $r_{\mathrm{u}} /\left\langle R^{2}\right\rangle^{1 / 2}$ ) on molecular weight $M$. In methyl ethyl ketone and cyclohexane this dependence is more pronounced because the coil structure in these solvents is denser. The chain length effect also explains the fact that for coils of

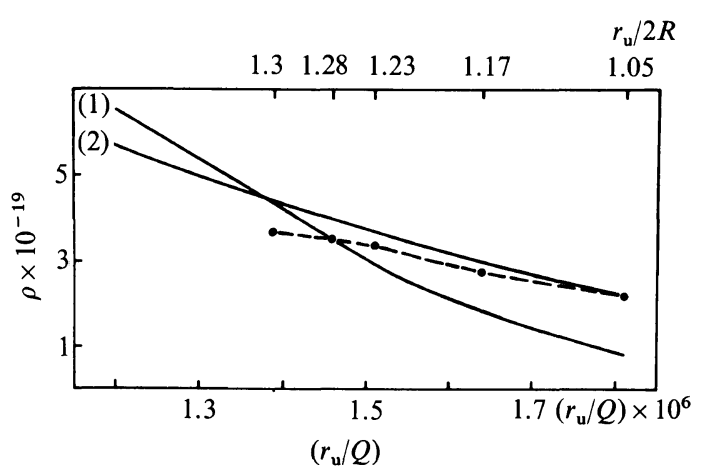

Figure 5. Points represent the density $\rho$ of units in the center of the zone of overlapping of two molecules as a function of $\left(r_{u} / 2\right)$ and relative permeability $\left(r_{u} / 2\left\langle R^{2}\right\rangle^{1 / 2}\right)$. From right to left: in benzene, methyl ethyl ketone and cyclohexane at $T=\theta+10^{\circ} \mathrm{C}, T=\theta$, and $T=\theta-10^{\circ} \mathrm{C}$. Curves represent the functions of unit distribution in 1) cyclohexane and 2) benzene; $\bar{M}_{w}=1.9 \times 10^{5}$.

low molecular weight exhibiting the highest density and lowest permeability, the $b_{\mathrm{o}} / V_{\mathrm{M}}$ ratio is close to 4.1 , the value characteristic of compact impermeable ellipsoids of revolution with the axes ratio of $2: 1 .^{11}$ With increasing $M$ (as mentioned above) the excluded volume $\left(b_{\mathrm{o}}\right)$ increases faster than the volume proper $\left(V_{\mathrm{M}}\right)$ and as a result the $b_{\mathrm{o}} / V_{\mathrm{M}}$ ratio becomes increasingly greater than the value of 4.1 . However, in a solvent in which coil swelling is relatively high (benzene) the values of $b_{\mathrm{o}}$ and $V_{\mathrm{M}}$ increase almost in the same direction as $M$, and the $b_{\mathrm{o}} / V_{\mathrm{M}}$ ratio varies only slightly. This situation shows that $b_{\mathrm{o}} / V_{\mathrm{M}}$ ratio is unsuitable as the measure of the shape asymmetry of macromolecules when they interpenetrate to some extent.

Afte this paper had alrealy been written, the authors became acquainted with a paper by Fujita et al. ${ }^{12}$ which doubtless can be included in the range of problems considered here. The data reported in ref 12 will be discussed by us in a future publication.

\section{REFERENCES}

1. V. E. Eskin, I. A. Baranovskaya, Vysokomol. Soedin. Ser. A, 19, 533 (1977).

2. I. A. Baranovskaya and V. E. Eskin, Vestnik of 


\section{E. Eskin, I. A. Baranovskaya, and U. S. Khudaiberdiev}

Leningrad University, No. 22, 159 (1969).

3. V. E. Eskin and I. A. Baranovskaya, Vysokomol. Soedin., Ser. A, 24, 2549 (1982).

4a. V. E Eskin., Vysokomol. Sodein., Ser. A, 26, 924 (1984).

4b. I. A. Baranovskaya, U. S. Khundaiberdiev, and V. E. Eskin, Vysokomol. Soedin., Ser. B. 26, 243 (1984).

5. B. H. Zimm, J. Chem, Phys., 16, 1093 (1948)

6. P. J. Flory, "Principles of Polymer Chemistry," Cornell Univ. Press, Ithaca, New York, 1953.

7. H. Yamakawa, "Modern Theory of Polymer
Solution," Harper \& Row, New York, N. Y., 1971, Chapter 4.

8. V. E. Eskin, Vysokomol. Soedin., Ser. B, 23, 725 (1981).

9. A. Einstein, Annal. Phys., 33, 1275 (1910).

10. P. Debye, J. Chem. Phys., 31, 680 (1959).

11. A. Ishihara and T. Hayashida , J. Phys. Soc. Jpn., 6, 46 (1951).

12. Z. Tong, S. Ohashi, Y. Einaga and H. Fujita, Polym. J., 15, 835 (1983). 